\title{
Hemangioendotelioma kaposiforme refractario en población pediátrica: reporte de caso y revisión de la literatura
}

\author{
Claudia P. Gómez-Villegas ${ }^{1}$, Camila Pérez-Téllez², Jorge Ochoa-Gaviria ${ }^{3}$ y Natalia Builes ${ }^{4 *}$ \\ ${ }^{1}$ Servicio UCl neonatal, Clínica La Sagrada Familia, Universidad del Quindío, Armenia; ${ }^{2}$ Pontificia Universidad Javeriana, Cali; ${ }^{3}$ Servicio de \\ Radiología, Hospital Pablo Tobón Uribe y Hospital Infantil San Vicente Fundación, Medellín; ${ }^{4}$ Servicio de Hematología Pediátrica, Hospital Pablo \\ Tobón Uribe, Medellín. Colombia
}

\begin{abstract}
Resumen
Introducción: El hemangioendotelioma kaposiforme (HEK) es un tumor vascular poco frecuente caracterizado por una invasión local agresiva y un síndrome de atrapamiento de plaquetas conocido como fenómeno de Kasabach-Merritt. Aunque muchos casos de HEK se tratan con éxito con control local o quimioterapia de baja intensidad, otros son resistentes y se cuenta con pocas opciones terapéuticas. El objetivo de este reporte es mostrar la experiencia del tratamiento con sirolimus por vía oral en un paciente pediátrico con HEK asociado a fenómeno de Kasabach-Merritt refractario al tratamiento de primera línea, quien mostró excelente respuesta al tratamiento. Caso clínico: Paciente de sexo masculino de 3 meses con un HEK refractario al manejo de primera línea (corticoides, propranolol, vincristina), sin posibilidad de hacer control local, por lo que se decide terapia combinada con sirolimus, presentando control local y resolución de la coagulopatía desde la primera semana de iniciado el manejo y con resolución de la malformación vascular después de 12 meses de seguimiento. Conclusiones: Aunque no existen pautas claras para el tratamiento del HEK refractario en la edad pediátrica, la evidencia actual demuestra que el sirolimus es un medicamento eficaz que puede ser considerado como opción terapéutica de primera línea en estos pacientes.
\end{abstract}

Palabras clave: Hemangioendotelioma kaposiforme. Sirolimus. Fenómeno de Kasabach-Merritt.

\section{Refractory kaposiforme hemangioendothelioma in the pediatric population: case report and literature review}

\begin{abstract}
Background: Kaposiform hemangioendothelioma (KHE) is a rare vascular tumor characterized by aggressive local invasion and a platelet entrapment syndrome known as the Kasabach-Merritt phenomenon. Although many cases of KHE are successfully treated with local control or low-intensity chemotherapy, some cases are often resistant, with few therapeutic options available. Here, we report a pediatric patient with KHE associated with Kasabach-Merritt phenomenon refractory to first-line treatment, who demonstrated excellent response to treatment. Case report: We present the case of a 3-month-old male patient with a KHE refractory to first-line treatment (vincristine, corticosteroids, propranolol), without possibility of local control treatment. Therefore, combined therapy with sirolimus was decided, presenting local control and resolution of the coagulopathy from the
\end{abstract}

Correspondencia:

*Natalia Builes

E-mail: natibui@ hotmail.com
Fecha de recepción: 08-10-2020

Fecha de aceptación: 20-11-2020

DOI: 10.24875/BMHIM.20000304
Disponible en internet: 23-07-2021

Bol Med Hosp Infant Mex. 2021;78(4):376-384

www.bmhim.com (http://creativecommons.org/licenses/by-nc-nd/4.0/) 
first week after starting the management and with resolution of vascular malformation after 12 months of follow-up. Conclusions: Although there are no clear guidelines for the treatment of refractory KHE in the pediatric population, current evidence demonstrate that sirolimus is an effective option that could be considered as a first-line treatment in such patients.

Keywords: Kaposiform hemangioendothelioma. Sirolimus. Kasabach-Merritt phenomenon.

\section{Introducción}

El hemangioendotelioma kaposiforme (HEK) es un tumor vascular muy poco común y agresivo que se manifiesta en el periodo neonatal o en la infancia con formas que pueden variar desde tumores vasculares cutáneos hasta grandes lesiones infiltrativas. Se ha reportado una incidencia aproximada de 0.7/100,000 niños por año ${ }^{1}$. Aproximadamente en el $50 \%$ de los casos, el HEK se encuentra presente al nacer; en el resto, se desarrolla después del periodo neonatal, con mayor frecuencia en el primer año de vida. Su localización habitual es en las extremidades y el tronco, pero se han descrito múltiples ubicaciones, incluso en el retroperitoneo. Las lesiones más agresivas se asocian a fenómeno de Kasabach-Merritt (FKM), una alteración hematológica grave, poco frecuente, caracterizada por trombocitopenia grave, anemia hemolítica microangiopática y la subsecuente coagulopatía de consumo asociada a tumores vasculares, concretamente al HEK 0 al angioma en racimo; además, presenta una alta mortalidad 2,3 .

A pesar de la gravedad de las posibles complicaciones, no se cuenta con pautas de manejo estandarizado en la población infantil ${ }^{4}$. Dentro de los esquemas terapéuticos disponibles se incluyen la escisión quirúrgica y múltiples tratamientos médicos, como esteroides sistémicos, vincristina, propranolol, antiplaquetarios, quimioterapia con agentes únicos o múltiples, embolización, escleroterapia y radioterapia, con eficacia variable ${ }^{5}$.

A pesar de las distintas opciones de tratamiento, la eficacia y la seguridad de estas no se han evaluado en ensayos aleatorizados ni en grandes estudios observacionales. La evidencia se limita a series de casos pequeñas, informes de casos únicos y la experiencia clínica.

Por otro lado, se sabe que la vía de la fosfatidilinositol 3-cinasa (PI3K/AKT) promueve el desarrollo vascular normal y la angiogénesis, y se ha demostrado que los trastornos que conducen a la activación inapropiada de esta vía llevan al crecimiento excesivo del tejido, asociado con anomalías vasculares. El sirolimus, un inhibidor de mTOR (mammalian target of rapamycin), integra las señales de la vía PI3K/AKT para coordinar el crecimiento y la proliferación celular adecuados mediante la regulación de la biogénesis ribosómica y la síntesis de proteínas. También actúa mejorando la señalización de mTOR, que a su vez aumenta la expresión del factor de crecimiento endotelial vascular, un regulador clave de la angiogénesis y la linfangiogénesis ${ }^{6}$.

Inicialmente, el sirolimus se administró como paliativo en un lactante con KHE y FKM que no había respondido a ningún algoritmo de tratamiento estándar? La justificación del tratamiento se basó en la activación de la vía PI3K/AKT/mTOR en la angiogénesis y la linfangiogénesis, así como en su uso en tumores, linfangioleiomiomatosis, esclerosis tuberosa y neurofibromas. Este paciente respondió al tratamiento con sirolimus, al igual que otros pacientes de alto riesgo ${ }^{8}$. A lo largo del tiempo, el sirolimus ha mostrado ser efectivo en el tratamiento de anomalías vasculares, evidenciando una importante actividad antiangiogénica ${ }^{9}$. Sin embargo, no hay datos para comparar las diferentes modalidades terapéuticas, lo que limita nuestra capacidad para decidir cuál podría ser el manejo inicial más apropiado.

El objetivo de este reporte fue mostrar la experiencia del tratamiento con sirolimus por vía oral en un paciente pediátrico con HEK asociado a FKM refractario al tratamiento de primera línea, con buena respuesta al medicamento. También se revisan los principales reportes que sustentan su uso.

\section{Caso clínico}

Paciente de sexo masculino, de 3 meses de edad, producto de primer embarazo, gemelar monocorial biamniótico de $36+3$ semanas de edad gestacional. En la semana 25 de gestación, por ecografía transabdominal se diagnosticó la presencia de una malformación vascular con características de hemangioma subcutáneo que comprometía el hemitórax izquierdo. Al nacimiento, el paciente se encontraba asintomático, por lo que se decidió manejo expectante. Sin embrago, a los 2 meses presentó progresión con múltiples pápulas solitarias: dos en el cráneo, una en el glúteo y los muslos, y una gran masa en el tórax y el miembro superior izquierdo. Además, aumentó el tamaño de la 


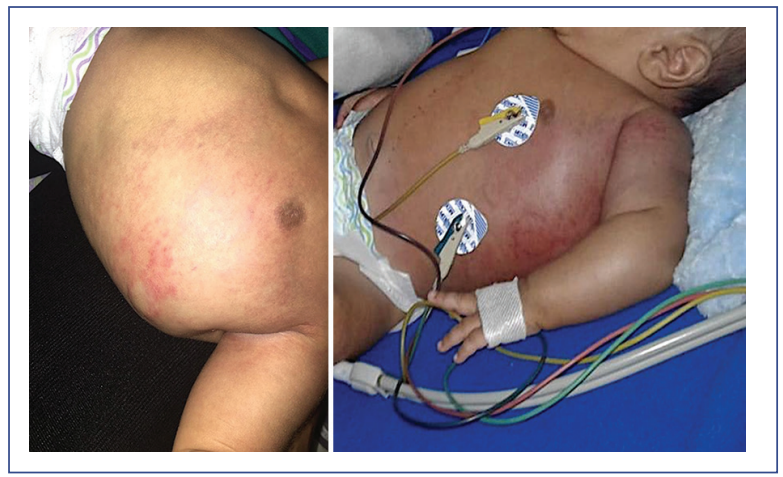

Figura 1. Aspecto inicial del tumor.

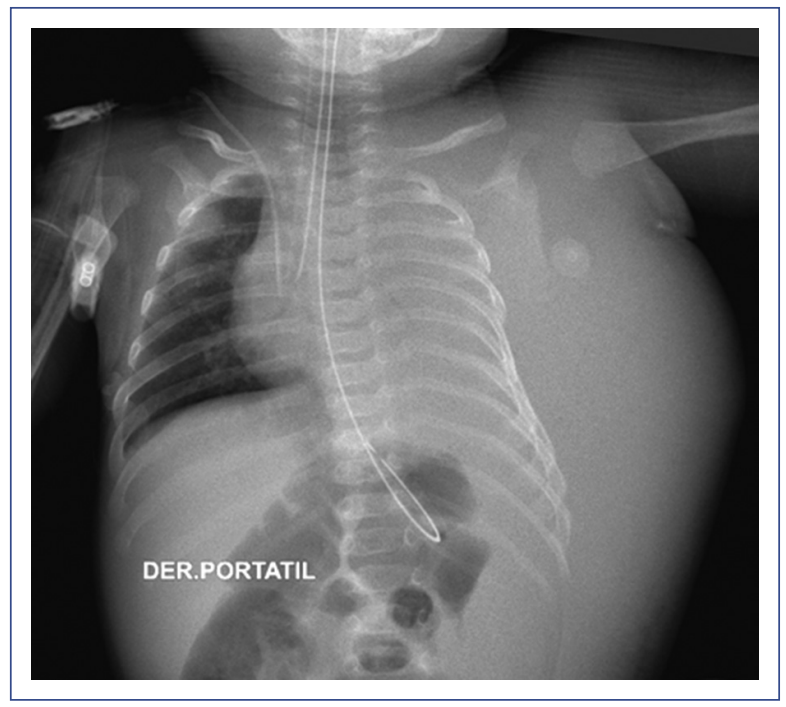

Figura 2. Radiografía de tórax en la que se observa el aumento de espesor de los tejidos blandos de la región toracoabdominal izquierda, sin evidente compromiso óseo. Atelectasia de pulmón izquierdo por intubación monobronquial.

lesión descrita en el hemitórax izquierdo, comprometiendo el tercio medio del hemiabdomen homolateral, el dorso y el miembro superior izquierdo. Adicionalmente, presentó cambios locales consistentes en eritema, calor y dolor a la palpación (Fig. 1).

Al ingreso a la institución se encontraron los siguientes hallazgos en los paraclínicos: hemoglobina $7.8 \mathrm{mg} / \mathrm{dl}$, plaquetas $7000 / \mathrm{mm}^{3}$, tiempo de protrombina $13.4 \mathrm{~s}$, tiempo de tromboplastina parcial activado $49.8 \mathrm{~s}$, fibrinógeno $116 \mathrm{mg} / \mathrm{dl}$ y dímero D 75,011 ng/ml. Se hospitalizó con sospecha de FKM para soporte y estudio por alto riesgo de hemorragia grave. Los criterios diagnósticos de los hemangiomas en la infancia

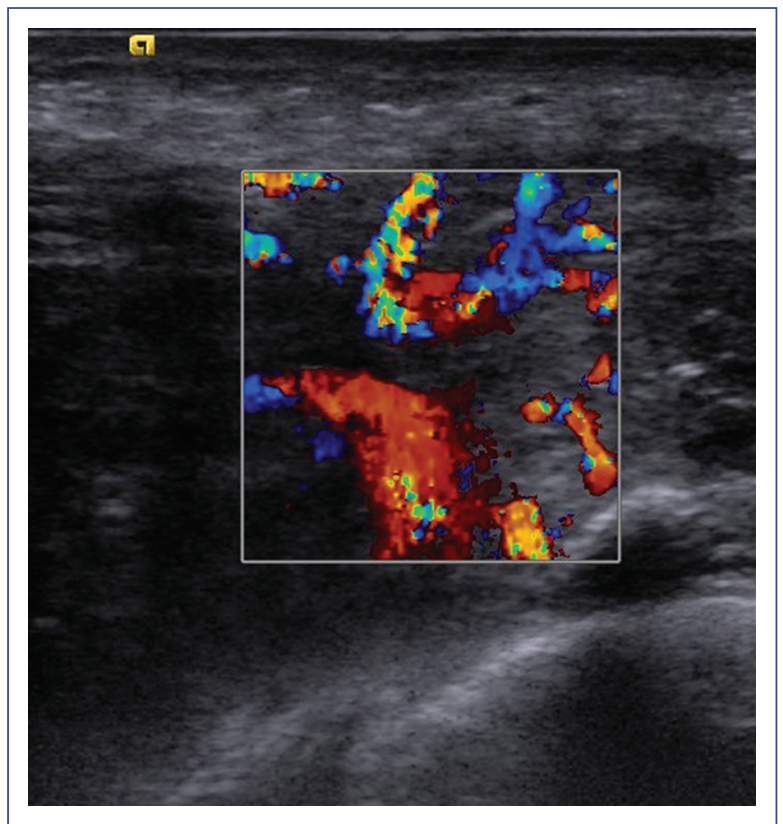

Figura 3. Ecografía Doppler de tejidos blandos. Lesión sólida irregular con vascularización prominente en su interior.

se establecen por la historia clínica, la exploración física y los estudios de imagen, como la radiografía simple, la ecografía, la tomografía computarizada, la resonancia magnética y la angiografía ${ }^{10}$.

El paciente fue diagnosticado durante la vida intrauterina con una ecografía obstétrica. El diagnóstico se confirmó considerando el antecedente prenatal, la exploración física y los estudios realizados durante la hospitalización. La radiografía de tórax, la ecografía Doppler y la angiorresonancia contrastada de aorta torácica y abdominal confirmaron la presencia de HEK (Figs. 2 a 4).

Se inició manejo con metilprednisolona a dosis de $1.6 \mathrm{mg} / \mathrm{kg} / \mathrm{día}$ y propranolol a dosis de $0.5 \mathrm{mg} / \mathrm{kg} / \mathrm{día}$ con posterior titulación hasta una dosis máxima de $3 \mathrm{mg} / \mathrm{kg}$. El paciente presentó un rápido deterioro clínico, con hemorragias mucocutáneas de difícil manejo, anemia hemolítica microangiopática y atelectasia pulmonar izquierda grave, por lo que requirió soporte ventilatorio invasivo. Se decidió adicionar al tratamiento vincristina semanal (dosis de $0.05 \mathrm{mg} / \mathrm{kg}$ ), completando 6 semanas, sin cambios, con alto requerimiento transfusional por persistencia del FKM. Considerando esta respuesta, se inició sirolimus a dosis de $1 \mathrm{mg} / \mathrm{m}^{2}$ cada 24 horas, con concentraciones séricas entre 10 y $13 \mathrm{ng} / \mathrm{ml}$ confirmadas 3 días después del inicio. A la semana de iniciado el sirolimus 


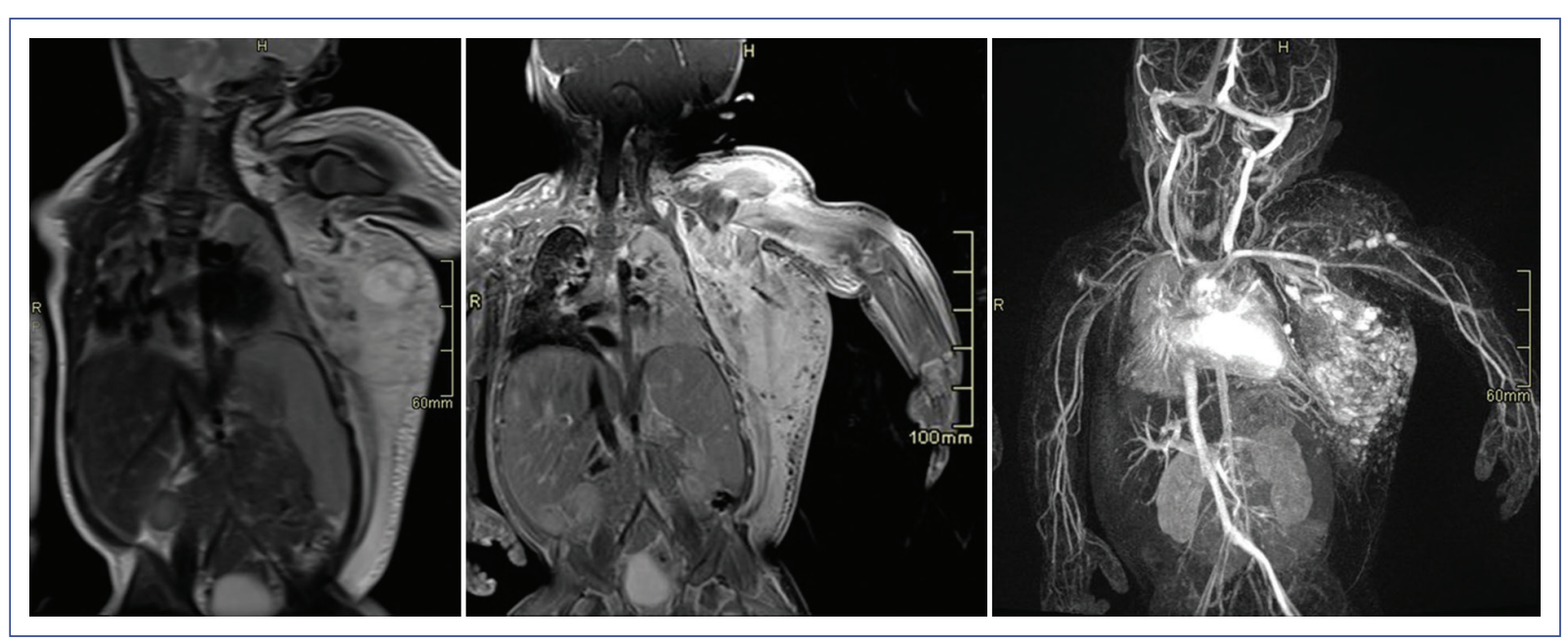

Figura 4. Resonancia contrastada (imágenes coronales en T2, T1 tras gadolinio y secuencia de angiorresonancia) en la que se observa un compromiso tumoral extenso dado por el aumento irregular y mal delimitado en el espesor de los tejidos blandos superficiales y profundos de la región toracoabdominal izquierda. Este compromiso se extiende a la región escapular y el miembro superior del mismo lado. El compromiso tumoral se realza de forma ávida y progresiva con el contraste, y se identifican estructuras vasculares prominentes que nutren la lesión.

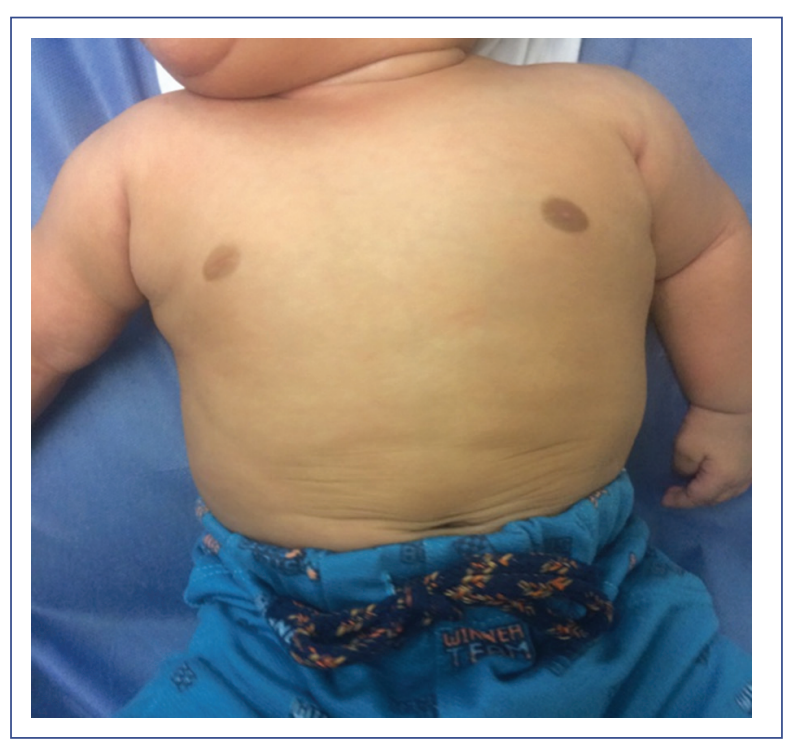

Figura 5. Aspecto del tumor a las 2 semanas de iniciado el sirolimus.

se evidenció una disminución objetiva del tamaño de la lesión (Fig. 5), así como un ascenso en la hemoglobina y en el conteo plaquetario (Fig. 6). Se decidió suspender el manejo con vincristina y esteroides, y continuar la monoterapia con sirolimus. Actualmente, el paciente ha completado 12 meses de tratamiento sin evidencia de coagulopatía y con resolución de la malformación vascular.

\section{Discusión}

El HEK es un tumor vascular raro, de características agresivas. Aproximadamente el $50 \%$ de las lesiones cutáneas son visibles o detectables al nacer, o se presentan durante la infancia. EI HEK se manifiesta como una masa de tejidos blandos elevada, con coloración purpúrica, consistencia dura y en ocasiones con telangiectasias $^{11,12}$. Afecta principalmente las extremidades y con menor frecuencia el tronco y la región cervicofacial; el retroperitoneo es la localización extracutánea más habitual, seguido de los músculos, los huesos y la cavidad torácica ${ }^{13}$. En el caso que se presenta, el diagnóstico se realizó prenatalmente y se manifestó con múltiples pápulas solitarias: dos en el cráneo, una en el glúteo y los muslos, y una gran masa en el tórax y el miembro superior izquierdo. La literatura refiere que el HEK congénito y el tamaño de la lesión se asocian con mayor riesgo de complicaciones ${ }^{1}$.

Por otro lado, el FKM es una complicación potencialmente mortal que puede ocurrir en el $70 \%$ de los pacientes. Se manifiesta con un aumento de tamaño del tumor y dolor. En la exploración física, la zona de la lesión se torna tensa y azul purpúrico. En las pruebas diagnósticas se evidencia trombocitopenia grave por el atrapamiento de las plaquetas en la lesión, anemia hemolítica microangiopática y coagulopatía de consumo con hipofibrinogenemia significativa ${ }^{14}$, motivo por el cual el paciente acudió a consulta y requirió 


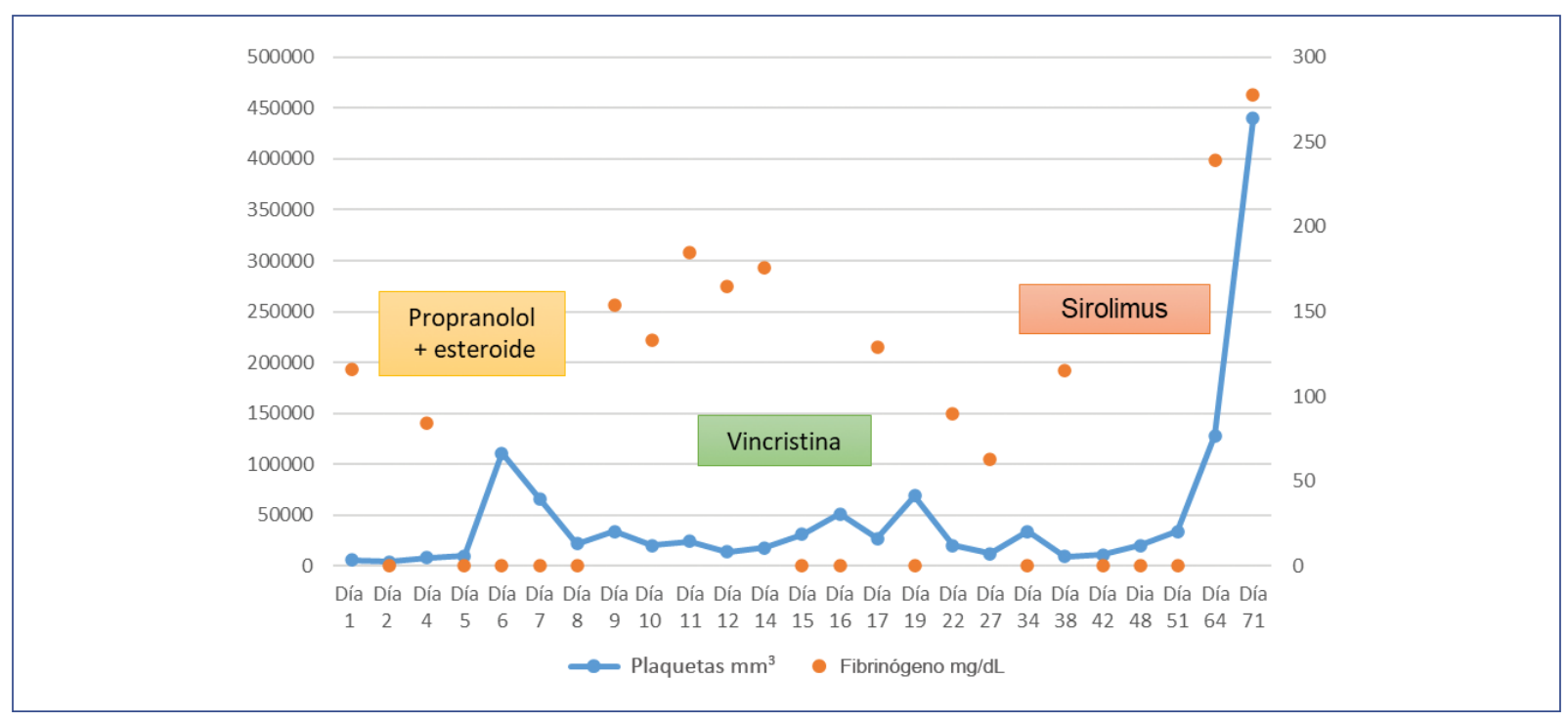

Figura 6. Comportamiento del conteo plaquetario y de los valores del fibrinógeno según cada modalidad terapéutica: propranolol más esteroide se inició el día 5, vincristina el día 14 y sirolimus el día 45.

hospitalización en la unidad de cuidados intensivos pediátricos.

El diagnóstico de HEK se basa en la combinación de las características clínicas, histológicas y de imagen, que son una valiosa ayuda dada la dificultad de realizar biopsias en algunos pacientes, como ocurrió en el caso aquí reportado. En los diferentes métodos de imagen, los HEK aparecen como tumores mal definidos que pueden comprometer los tejidos blandos superficiales o profundos ${ }^{15}$. Por ecografía, se observan como masas mal definidas, vascularizadas, con estructuras de baja y alta resistencia, y ecogenicidad heterogénea ${ }^{16}$. Al ser estos tumores infiltrativos, la resonancia magnética usualmente se utiliza para caracterizar la extensión de la lesión. En ella, los HEK se caracterizan por ser lesiones isointensas o hipointensas en las secuencias $\mathrm{T} 1 \mathrm{e}$ hiperintensas en las secuencias T2. Posterior al contraste, son tumores hipervasculares con realce heterogéneo y compromiso de múltiples planos de tejido blando. La grasa alrededor del tumor puede estar edematizada en el momento del diagnóstico ${ }^{13,17-19}$.

Aún no existen pautas claras para el tratamiento del HEK debido a la heterogeneidad de su presentación. En el año 2013, un panel multidisciplinario de expertos de los Estados Unidos y de Canadá propusieron unas guías para el tratamiento del HEK con complicaciones asociadas, basándose en la revisión de la evidencia disponible, la opinión de expertos y la experiencia clínica. Se recomendaron los corticoides sistémicos y la vincristina como tratamiento de primera línea. Sin embargo, el enfoque terapéutico debe ser individualizado según el tamaño, la ubicación, la presencia de síntomas o compromiso funcional, y la presencia o ausencia de trombocitopenia y coagulopatía ${ }^{20}$.

Existen reportes de casos y un ensayo de fase II con sirolimus en monoterapia o combinado con corticoides sistémicos para casos refractarios a terapias previas, que respaldan su uso como primera línea en pacientes con HEK complicado con o sin FKM, porque puede administrarse por vía oral y porque la coagulopatía mejora más rápido ${ }^{8,9}$.

A continuación se resumen las recomendaciones encontradas en la literatura sobre el tratamiento:

- La resección quirúrgica es un tratamiento definitivo de elección en tumores bien definidos y localizados. La resección durante la fase activa de FKM no es aconsejable. La edad del paciente, el tamaño y la vascularización de los tumores son factores de riesgo para la presencia de hemorragias, lesiones iatrogénicas y empeoramiento de la coagulopatía. Por este motivo, la cirugía rara vez es una opción para HEK extensos y para pacientes en quienes la cirugía dará como resultado un deterioro funcional significativo. En el presente caso, por la extensión del tumor y la corta edad del paciente, no estaba indicado un procedimiento intervencionista ${ }^{21}$.

- El objetivo del tratamiento médico es la mejoría de los síntomas y de la coagulopatía, y disminuir el tamaño de la masa tumoral. Las opciones son las siguientes: 
- Los corticoesteroides sistémicos se utilizan como tratamiento de primera línea en los casos de HEK sin $\mathrm{FKM}^{22}$. Se puede iniciar prednisolona a dosis de $2 \mathrm{mg} / \mathrm{kg} / \mathrm{día}$ por vía oral o metilprednisolona a dosis de $1.6 \mathrm{mg} / \mathrm{kg} /$ día por vía intravenosa. La estabilización del recuento plaquetario puede ocurrir en 1-2 días, pero la respuesta sostenida es variable. La mayoría de los pacientes no mejoran con los corticoesteroides solos (la tasa de respuesta global es del 10-27\%). Si se observa un aumento en el recuento de plaquetas en 102 semanas, se inicia el descenso de los corticoides. Si no hay respuesta o hay recaída de la trombocitopenia con el descenso de la dosis, se recomienda el tratamiento con un segundo agente, que puede ser vincristina o sirolimus. El uso de corticoesteroides a largo plazo presenta múltiples efectos secundarios no deseados ${ }^{5,23}$.

- El propranolol es un antagonista beta-adrenérgico no selectivo. Se administra a dosis de $2-3 \mathrm{mg} / \mathrm{kg} / \mathrm{día}$ por vía oral. Se ha sugerido para el tratamiento del HEK por la respuesta eficaz en el tratamiento de los hemangiomas infantiles. Sin embargo, en una serie de casos solo se obtuvo respuesta en el $36 \%$ de los pacientes ${ }^{24}$. No se recomienda como monoterapia en pacientes con $\mathrm{FKM}^{4}$. En ocasiones se ha usado en combinación con vincristina ${ }^{25}$. Al ingreso, el paciente de este caso inició tratamiento con metilprednisolona a dosis de $1.6 \mathrm{mg} / \mathrm{kg} /$ día y propranolol a dosis de $3 \mathrm{mg} / \mathrm{kg} / \mathrm{día}$, sin mejoría.

- La vincristina es un agente de quimioterapia que promueve la apoptosis endotelial. Se administra a dosis semanales de $0.025-0.05 \mathrm{mg} / \mathrm{kg}$ para lactantes con peso $<10 \mathrm{~kg}$ y de $1.0-1.5 \mathrm{mg} / \mathrm{m}^{2}$ para lactantes con peso $>10 \mathrm{~kg}$, durante 2 meses, por vía intravenosa mediante acceso venoso central. Es la terapia de primera línea en combinación con corticoesteroides sistémicos, y la respuesta es del $72 \%{ }^{26}$. Si se observa una buena respuesta clínica y se estabiliza el estado hematológico del paciente, el objetivo es disminuir los corticoesteroides durante 4 u 8 semanas hasta suspenderlos. En una serie de casos, el tiempo promedio para el aumento del recuento plaquetario fue de 2 meses, y el tiempo promedio para disminuir el tamaño del tumor fue de 4 meses. Si se observa respuesta (coagulopatía, regresión completa o reducción del tamaño del tumor), el intervalo de administración puede aumentarse a 2 semanas durante 2 meses y luego cada 3 semanas durante 2 meses $^{27}$. Los efectos adversos de la vincristina incluyen polineuropatía periférica, pérdida de reflejos tendinosos y disfunción autonómica abdominal ${ }^{13}$. En el paciente reportado, tras evaluar la falta de respuesta al tratamiento se inició vincristina en combinación con prednisolona, a dosis semanal. Inicialmente presentó mejoría, pero luego de nuevo aumentó el tamaño del tumor y descendió el recuento de plaquetas.

- El sirolimus es un inmunosupresor e inhibidor de la rapamicina (mTOR). Es una serina/treonina cinasa regulada por la fosfoinositida-3-cinasa que afecta el crecimiento celular y la angiogénesis, y también es un potente inmunosupresor y un medicamento antitumoral. Se ha sugerido como una opción de tratamiento para las anomalías vasculares y los tumores complicados en niños, incluidos HEK y FKM. La dosis recomendada es de 0.8-1 mg/ $/ \mathrm{m}^{2}$ por toma, dos veces al día, por vía oral. Se debe hacer seguimiento monitorizando las concentraciones séricas del sirolimus, que no deben ser $>10-13 \mathrm{ng} / \mathrm{ml}^{28}$. El paciente aquí reportado no presentó mejoría con las terapias de primera línea, por lo que se procedió al manejo con sirolimus, inicialmente de forma combinada, pero paulatinamente se logró la disminución del esteroide, previniendo así complicaciones derivadas por su uso a largo plazo.

Se realizó una revisión de la literatura de los últimos 10 años utilizando las bases de datos PubMed y EMBASE, con los siguientes términos para la búsqueda: hemangioendothelioma (HEK) y sirolimus. Se incluyeron reportes de casos o series de casos en los que se reportara el uso de sirolimus, particularmente aquellos que hacían referencia a la posología, el tiempo de tratamiento, los efectos adversos y la respuesta al tratamiento. Schmid, et al. ${ }^{5}$ realizaron un reporte de caso con revisión de la literatura entre 1993 y 2017, que reveló un total de 105 publicaciones sobre HEK en menores de 21 años, con un total de 231 pacientes. De estos, el $79 \%$ presentaron FKM, y de estos, a su vez, el $97 \%$ requirieron manejo con sirolimus. En las bases de datos PubMed, EBSCO y Ovid no se encontró ningún reporte sobre casos de HEK en Colombia; existen reportes de casos aislados en Chile, Perú y México.

En todos los casos encontrados, los pacientes presentaron respuesta completa con un tiempo de máximo de tratamiento de 24 meses. Las concentraciones séricas fueron entre 5 y $15 \mathrm{ng} / \mathrm{ml}$ y las dosis utilizadas fueron entre 0.4 y $1.5 \mathrm{mg} / \mathrm{m}^{2}$ cada 12 horas. Las principales ventajas del uso de sirolimus incluyen la administración por vía oral y la resolución más rápida de la coagulopatía, en comparación con la combinación de 
Tabla 1. Reportes de casos uso de sirolimus en hemangioendoteliomas kaposiformes

\begin{tabular}{|c|c|c|c|c|c|c|}
\hline Autores & Año & $\begin{array}{l}\text { Número de } \\
\text { pacientes }\end{array}$ & Dosis & Respuesta & $\begin{array}{l}\text { Tiempo de } \\
\text { tratamiento }\end{array}$ & Efectos adversos \\
\hline $\begin{array}{l}\text { Freixo, } \\
\text { et al. } .^{29}\end{array}$ & 2020 & 121 & $\begin{array}{l}\text { Dosis de } 0.8 \mathrm{mg} / \mathrm{m}^{2} \\
\text { cada } 12 \text { horas } \\
\text { ajustada con } \\
\text { concentraciones } \\
\text { séricas de } \\
5-15 \mathrm{ng} / \mathrm{ml}\end{array}$ & $\begin{array}{l}\text { El } 95.5 \% \text { de los } \\
\text { pacientes mejoraron } \\
\text { clínicamente. En el } 93 \% \\
\text { se normalizó la } \\
\text { coagulopatía. Se } \\
\text { observó mejoría en } \\
\text { promedio en } 13.7 \text { días }\end{array}$ & $\begin{array}{l}\text { La mediana de } \\
\text { duración del } \\
\text { tratamiento fue } \\
\text { de } 8.5 \text { meses } \\
\text { (0.23-216 } \\
\text { meses). } \\
\text { Respuesta } \\
\text { clínica entre } 7 \\
\text { y } 21 \text { días }\end{array}$ & Ninguno reportado \\
\hline $\begin{array}{l}\text { Guerrero- } \\
\text { Padilla, } \\
\text { et al. }\end{array}$ & 2019 & 1 & $\begin{array}{l}\text { Dosis de } 0.8 \mathrm{mg} / \mathrm{m}^{2} \\
\text { cada } 12 \text { horas con } \\
\text { concentraciones } \\
\text { séricas de } 10-18 \\
\mathrm{ng} / \mathrm{ml}\end{array}$ & $\begin{array}{l}\text { Respuesta adecuada a } \\
\text { la segunda semana, con } \\
\text { disminución paulatina }\end{array}$ & 12 meses & $\begin{array}{l}\text { Ninguno } \\
\text { reportado. } \\
\text { Recomiendan } \\
\text { profilaxis para } \\
\text { Pneumocystis } \\
\text { jirovecii }\end{array}$ \\
\hline $\begin{array}{l}\text { Mariani, } \\
\text { et al. }^{31}\end{array}$ & 2019 & 1 & $\begin{array}{l}\text { Dosis de } 0.4 \mathrm{mg} / \mathrm{m}^{2} \\
\text { por dosis, dos } \\
\text { veces al día }\end{array}$ & Completa & No disponible & Ninguno reportado \\
\hline $\begin{array}{l}\text { Wang, } \\
\text { et al. } .^{32}\end{array}$ & 2018 & 8 & $\begin{array}{l}\text { Dosis de } 0.05 \mathrm{mg} / \mathrm{kg} \\
\text { dos veces al día }\end{array}$ & Completa & $\begin{array}{l}12 \text { a } 79 \\
\text { semanas }\end{array}$ & Mucositis \\
\hline $\begin{array}{l}\text { Chinello, } \\
\text { et al. }{ }^{33}\end{array}$ & 2018 & $\begin{array}{l}\text { Reporte de un } \\
\text { caso + revisión: } \\
89 \text { pacientes con } \\
\text { HEK y } 15 \\
\text { pacientes } \\
\text { tratados con } \\
\text { sirolimus como } \\
\text { terapia de } \\
\text { segunda línea }\end{array}$ & $\begin{array}{l}\text { Dosis de } 3 \mathrm{mg} / \mathrm{m}^{2} \\
\text { con } \\
\text { concentraciones } \\
\text { séricas de } \\
7-10 \mathrm{ng} / \mathrm{ml}\end{array}$ & $\begin{array}{l}\text { Mejoría completa. } \\
\text { Presentó mejoría del } \\
\text { recuento de plaquetas } \\
\text { después de } 15 \text { días de } \\
\text { tratamiento y las } \\
\text { plaquetas se } \\
\text { normalizaron después } \\
\text { de } 4 \text { meses. Además, } \\
\text { presentó reducción } \\
\text { progresiva del HEK. En } \\
\text { los pacientes de la } \\
\text { revisión de la literatura, } \\
\text { la terapia a largo plazo } \\
\text { con sirolimus demostró } \\
\text { ser efectiva para } \\
\text { controlar la enfermedad }\end{array}$ & 20 meses & Ninguno reportado \\
\hline $\begin{array}{l}\text { Cashell, } \\
\text { et al. }\end{array}$ & 2018 & 1 & $\begin{array}{l}\text { Dosis de } 0.8 \mathrm{mg} / \mathrm{m}^{2} \\
\text { por dosis, dos } \\
\text { veces al día, con } \\
\text { concentraciones } \\
\text { séricas de } 10-15 \\
\mathrm{ng} / \mathrm{ml}\end{array}$ & $\begin{array}{l}\text { Completa. Luego de } 2 \\
\text { semanas de tratamiento, } \\
\text { se logró el descenso del } \\
15 \% \text { de los } \\
\text { corticoesteroides cada } 2 \\
\text { semanas. Al alcanzar la } \\
\text { concentración sérica se } \\
\text { suspendieron } \\
\text { progresivamente las } \\
\text { transfusiones a los } 19 \\
\text { días. A las } 7 \text { semanas, } \\
\text { disminuyó clínicamente } \\
\text { el tamaño del } \\
\text { hemangioma. La } \\
\text { vincristina se suspendió } \\
\text { a las } 21 \text { semanas }\end{array}$ & 12 meses & $\begin{array}{l}\text { Mucositis, que se } \\
\text { resolvió al } \\
\text { optimizar las } \\
\text { concentraciones } \\
\text { séricas }\end{array}$ \\
\hline $\begin{array}{l}\text { MacFarland, } \\
\text { et al. }{ }^{35}\end{array}$ & 2017 & 1 & $\begin{array}{l}\text { Dosis de } 3 \mathrm{mg} / \mathrm{m}^{2} \\
\text { al día }\end{array}$ & Completa & 8 meses & Ninguno reportado \\
\hline
\end{tabular}


Tabla 1. Reportes de casos uso de sirolimus en hemangioendoteliomas kaposiformes (continuación)

\begin{tabular}{|c|c|c|c|c|c|c|}
\hline Autores & Año & $\begin{array}{l}\text { Número de } \\
\text { pacientes }\end{array}$ & Dosis & Respuesta & $\begin{array}{l}\text { Tiempo de } \\
\text { tratamiento }\end{array}$ & Efectos adversos \\
\hline $\begin{array}{l}\text { Rodríguez, } \\
\text { et al. } .^{36}\end{array}$ & 2013 & 2 & $\begin{array}{l}\text { Dosis de } 0.8 \mathrm{mg} / \mathrm{m}^{2} \\
\text { por dosis, dos } \\
\text { veces al día, con } \\
\text { concentraciones } \\
\text { séricas de } \\
5-15 \mathrm{ng} / \mathrm{ml}\end{array}$ & $\begin{array}{l}\text { Completa. Se observó } \\
\text { normalización de } \\
\text { pruebas hematológicos, } \\
\text { desaparición del dolor y } \\
\text { disminución paulatina } \\
\text { de la masa tumoral }\end{array}$ & $\begin{array}{l}\text { Mejoría a los } 3 \\
\text { meses. } \\
\text { Tratamiento } \\
\text { entre } 12 \text { y } 24 \\
\text { meses }\end{array}$ & - \\
\hline $\begin{array}{l}\text { Jahnel, } \\
\text { et al. }{ }^{37}\end{array}$ & 2012 & 1 & $\begin{array}{l}\text { Dosis de } 0.1 \mathrm{mg} / \mathrm{kg} \\
\text { al día, con } \\
\text { concentraciones } \\
\text { séricas de } \\
10-15 \mathrm{ng} / \mathrm{ml}\end{array}$ & $\begin{array}{l}\text { Después de } 4 \text { semanas, } \\
\text { los índices de } \\
\text { coagulación fueron } \\
\text { normales. Se retiró el } \\
\text { esteroide. A las } 11 \\
\text { semanas se suspendió } \\
\text { la vincristina }\end{array}$ & 33 semanas & $\begin{array}{l}\text { Mínima lesión: } \\
\text { decoloración } \\
\text { azul-rojiza }\end{array}$ \\
\hline $\begin{array}{l}\text { Hammill, } \\
\text { et al. }{ }^{7}\end{array}$ & 2011 & 6 & $\begin{array}{l}\text { Dosis de } 0.8 \mathrm{mg} / \mathrm{m}^{2} \\
\text { cada } 12 \text { horas, con } \\
\text { concentraciones } \\
\text { séricas de } \\
10-15 \mathrm{ng} / \mathrm{ml}\end{array}$ & $\begin{array}{l}\text { Completa. Mejoría entre } \\
1 \text { y } 2 \text { semanas de } \\
\text { iniciado el tratamiento }\end{array}$ & $\begin{array}{l}\text { El tiempo de } \\
\text { tratamiento } \\
\text { varió entre } 2 \text { y } \\
28 \text { meses, con } \\
\text { un promedio de } \\
22 \text { meses }\end{array}$ & Ninguno reportado \\
\hline
\end{tabular}

HEK: hemangioendotelioma kaposiforme.

vincristina y corticoesteroides. Los efectos secundarios del sirolimus informados con mayor frecuencia son mucositis oral, dislipidemia, leucocitopenia, síntomas gastrointestinales y erupción cutánea o eccema. No hay reportes de afectos adversos graves. Aunque se obtuvo una respuesta positiva con toxicidad limitada en los casos publicados, no existe una estandarización de los criterios de respuesta ni de toxicidad (Tabla 1) 29-37. $^{2}$.

Los HEK son tumores vasculares infrecuentes y localmente agresivos en la edad pediátrica' ${ }^{1}$. Aún no se dispone de pautas claras para su tratamiento debido a la heterogeneidad de su presentación clínica, la cual puede tener un comportamiento agresivo en la edad pediátrica que requiera intervenciones terapéuticas rápidas para mitigar las complicaciones asociadas. La evidencia actual demuestra que el sirolimus es un medicamento eficaz, con mínimos efectos adversos y fácil de administrar, que puede ser considerado como opción de tratamiento inicial.

\section{Responsabilidades éticas}

Protección de personas y animales. Los autores declaran que para esta investigación no se han realizado experimentos en seres humanos ni en animales.

Confidencialidad de los datos. Los autores declaran que han seguido los protocolos de su centro de trabajo sobre la publicación de datos de pacientes.
Derecho a la privacidad y consentimiento informado. Los autores han obtenido el consentimiento informado de los pacientes o individuos referidos en el artículo. Este documento obra en poder del autor de correspondencia.

\section{Conflicto de intereses}

Los autores declaran no tener ningún conflicto de intereses, particularmente con el uso del sirolimus.

\section{Financiamiento}

Ninguno.

\section{Bibliografía}

1. Ji Y, Chen S, Yang K, Xia C, Li L. Kaposiform hemangioendothelioma: current knowledge and future perspectives. Orphanet J Rare Dis. 2020;15:39.

2. Bruder E, Alaggio R, Kozakewich HP, Jundt G, Dehner LP, Coffin CM. Vascular and perivascular lesions of skin and soft tissues in children and adolescents. Pediatr Dev Pathol. 2012;15:26-61.

3. Sarkar M, Mulliken JB, Kozakewich HP, Robertson RL, Burrows PE. Thrombocytopenic coagulopathy (Kasabach-Merritt phenomenon) is associated with Kaposiform hemangioendothelioma and not with common infantile hemangioma. Plast Reconstr Surg. 1997;100:1377-86.

4. Tlougan BE, Lee MT, Drolet BA, Frieden IJ, Adams DM, Garzon MC. Medical management of tumors associated with Kasabach-Merritt phenomenon: an expert survey. J Pediatr Hematol Oncol. 2013;35:618-22.

5. Schmid I, Klenk AK, Sparber-Sauer M, Koscielniak E, Maxwell R, Häberle B. Kaposiform hemangioendothelioma in children: a benign vascular tumor with multiple treatment options. World J Pediatr. 2018;14:322-9.

6. Tee AR, Blenis J. mTOR, translational control and human disease. Semin Cell Dev Biol. 2005;16:29-37. 
7. Hammill AM, Wentzel M, Gupta A, Nelson S, Lucky A, Elluru R, et al Sirolimus for the treatment of complicated vascular anomalies in children. Pediatr Blood Cancer. 2011;57:1018-24.

8. Adams DM, Trenor CC $3^{\text {rd }}$, Hammill AM, Vinks AA, Patel MN, Chaudry G et al. Efficacy and safety of sirolimus in the treatment of complicated vascular anomalies. Pediatrics. 2016;137:e20153257.

9. Stacchiotti S, Provenzano S, Dagrada G, Negri T, Brich S, Basso U, et al. Sirolimus in advanced epithelioid hemangioendothelioma: a retrospective case-series analysis from the Italian Rare Cancer Network Database. Ann Surg Oncol. 2016;23:2735-44.

10. Burrows PE, Laor T, Paltiel H, Robertson RL. Diagnostic imaging in the evaluation of vascular birthmarks. Dermatol Clin. 1998;16:455-88.

11. Ji Y, Yang K, Peng S, Chen S, Xiang B, Xu Z, et al. Kaposiform haemangioendothelioma: clinical features, complications and risk factors for Kasabach-Merritt phenomenon. Br J Dermatol. 2018;179:457-63.

12. Zukerberg LR, Nickoloff BJ, Weiss SW. Kaposiform hemangioendothelioma of infancy and childhood. An aggressive neoplasm associated with Kasabach-Merritt syndrome and lymphangiomatosis. Am J Surg Pathol. 1993;17:321-8.

13. Croteau SE, Liang MG, Kozakewich HP, Alomari Al, Fishman SJ, Mulliken JB, et al. Kaposiform hemangioendothelioma: atypical features and risks of Kasabach-Merritt phenomenon in 107 referrals. J Pediatr. 2013;162:142-7.

14. Kelly M. Kasabach-Merritt phenomenon. Pediatr Clin North Am 2010;57:1085-9.

15. Lyons LL, North PE, Mac-Moune Lai F, Stoler MH, Folpe AL, Weiss SW Kaposiform hemangioendothelioma: a study of 33 cases emphasizing its pathologic, immunophenotypic, and biologic uniqueness from juvenile hemangioma. Am J Surg Pathol. 2004;28:559-68.

16. Restrepo R, Francavilla ML, Mas R, Lee EY. Up-to-date practical imaging evaluation of neonatal soft tissue tumors: what radiologist need to know. Am J Roentgenol. 2017;209:195-204.

17. Malhotra Y, Yang CS, McNamara J, Antaya RJ. Congenital kaposiform hemangioendothelioma with Kasabach-Merritt phenomenon successfully treated with low-dose radiation therapy. Pediatr Dermatol. 2014;31:595-8.

18. Arnold R, Chaudry G. Diagnostic imaging of vascular anomalies. Clin Plast Surg. 2011;38:21-9.

19. Drolet BA, Trenor CC $3^{\text {rd }}$, Brandão LR, Chiu YE, Chun RH, Dasgupta R et al. Consensus-derived practice standards plan for complicated kaposiform hemangioendothelioma. J Pediatr. 2013;163:285-91.

20. Adams D, Trenor C, Ricci K et al. Phase II study of sirolimus and complicated vascular anomalies: long-term outcomes in kaposiform hemangioendothelioma. $22^{\text {nd }}$ International Workshop of the International Society for the Study of Vascular Anomalies. Amsterdam; 2018.

21. Gruman A, Liang MG, Mulliken JB, Fishman SJ, Burrows PE, Kozakewich HP, et al. Kaposiform hemangioendothelioma without Kasabach-Merritt phenomenon. J Am Acad Dermatol. 2005;52:616-22.

22. Ryan C, Price V, John P, Mahant S, Baruchel S, Brandão L, et al. Kasabach-Merritt phenomenon: a single centre experience. Eur J Haematol. 2010;84:97-104.

23. Özsoylu Ş. Megadose methylprednisolone for Kasabach-Merritt syndrome. Pediatr Hematol Oncol. 1999;16:373-4.
24. Chiu YE, Drolet BA, Blei F, Carcao M, Fangusaro J, Kelly ME, et al. Variable response to propranolol treatment of kaposiform hemangioendothelioma, tufted angioma, and Kasabach-Merritt phenomenon. Pediatr Blood Cancer. 2012;59:934-8.

25. Hermans DJ, van Beynum IM, van der Vijver RJ, Kool LJ, de Blaauw I, van der Vleuten CJ. Kaposiform hemangioendothelioma with Kasabach-Merritt syndrome: a new indication for propranolol treatment. J Pediatr Hematol Oncol. 2011;33:e171-3.

26. Wang Z, Li K, Yao W, Dong K, Xiao X, Zheng S. Steroid-resistant kaposiform hemangioendothelioma: a retrospective study of 37 patients treated with vincristine and long-term follow-up. Pediatr Blood Cancer. 2015;62:577-80.

27. Haisley-Royster C, Enjolras O, Frieden IJ, Garzon M, Lee M, Oranje A, et al. Kasabach-Merritt phenomenon: a retrospective study of treatment with vincristine. J Pediatr Hematol Oncol. 2002;24:459-62. [Published correction appears in J Pediatr Hematol Oncol 2002;24:794].

28. Blatt J, Stavas J, Moats-Staats B, Woosley J, Morrell DS. Treatment of childhood kaposiform hemangioendothelioma with sirolimus. Pediatr Blood Cancer. 2010;55:1396-8.

29. Freixo C, Ferreira V, Martins J, Almeida R, Caldeira D, Rosa M, et al. Efficacy and safety of sirolimus in the treatment of vascular anomalies: a systematic review. J Vasc Surg. 2020;71:318-27.

30. Guerrero-Padilla D, Tantaleán-Da Fieno J, Velásquez-Valderrama F, León-Paredes R. Thoracic kaposiform hemangioendothelioma with a good response to sirolimus. Case report and literature review. Acta Med Peru. 2019;36:124-8.

31. Mariani LG, Schmitt IR, García CD, Kiszewski AE. Low dose sirolimus treatment for refractory tufted angioma and congenital kaposiform hemangioendothelioma, both with Kasabach-Merritt phenomenon. Pediatr Blood Cancer. 2019;66:e27810.

32. Wang H, Guo X, Duan Y, Zheng B, Gao Y. Sirolimus as initial therapy for kaposiform hemangioendothelioma and tufted angioma. Pediatr Dermatol. 2018;35:635-8.

33. Chinello M, Di Carlo D, Olivieri F, Balter R, De Mortoli M, Vitale V, et al. Successful management of kaposiform hemangioendothelioma with longterm sirolimus treatment: a case report and review of the literature. Mediterr J Hematol Infect Dis. 2018;10:e2018043.

34. Cashell J, Smink GM, Helm K, Xavier F. Kaposiform hemangioendothelioma with Kasabach-Merritt phenomenon in an infant: successful treatment with prednisolone, vincristine, and addition of sirolimus. Pediatr Blood Cancer. 2018;65:e27305

35. MacFarland SP, Sullivan LM, States LJ, Bailey LC, Balamuth NJ, Womer RB, et al. Management of refractory pediatric kaposiform hemangioendothelioma with sirolimus and aspirin. J Pediatr Hematol Oncol. 2018;40:e239-42.

36. Rodríguez ZN, Benavides JP. Sirolimus (rapamicina) en pacientes con hemangioendotelioma kaposiforme: caso clínico. Rev Chil Pediatr. 2013;84:537-44

37. Jahnel J, Lackner H, Reiterer F, Urlesberger B, Urban C. Kaposiform hemangioendothelioma with Kasabach-Merritt phenomenon: from vincristine to sirolimus. Klin Padiatr. 2012;224:395-7. 